VS20-P03

\section{Scope and limitations on refinement strategy. service crystallographer's publishing woes}

\author{
Arkady Ellern ${ }^{1}$ \\ 1. Chemistry Department. Iowa State University, Ames, Iowa, United \\ States of America \\ email: ellern@iastate.edu
}

Small molecule X-Ray structure determination can be considered as a three step process where each step is dependent the previous one.

Data collection. All special techniques (unstable samples, low temperature, high pressure experiments etc.) were well established many years ago. The revolution in computing during the last two decades speed up this step significantly as modern diffractometers with power radiation sources can collect full-sphere data with high redundancy in a few hours. As a result this step nowadays is absolutely routine in all specialized facilities

Structure Solution/Refinement. "Black-box", like Bruker EXPRESSO, obtains good results for simple samples by users without sufficient background in symmetry, crystallography, diffraction, refinement and even chemistry. However, in real life the majority of structures must be resolved unambiguously only by experienced professionals to consider the best route and final point of analysis, which is not always obvious and beyond a reasonable doubts.

As an example, giant spherical keplerate cluster structures like $\left\{\mathrm{Mo}_{72} \mathrm{~V}_{30}\right\}$ require a lot of restrains to model the disorder during the refinement to hold outer and inner ligands at reasonable positions and with acceptable temperature factors. The concomitant difficulties are: the split positions for metal core, the huge voids filled with diffused water solvent, uncertainties in the valence state of metals and occupancy of counter-ions. Where is the limit of data "massaging" and "fixing" on the way of supporting our model of choice? Where should we stop?

Majority of customers use X-Ray structure determination for the qualitative and quantitative analysis which means they are fully satisfied with the connectivity table to assure the synthetic path and the proof of obtaining the target substance. How far should we go with such structures? The refinement for the simple metallo-organic complex can illustrate how stepwise split of disordered benzene solvent position influence the geometrical and thermal parameters of the main cluster.

Publication. Can we trust old X-ray structural results even though they can hardly pass the new strict requirements for publication? How many different expert opinions can we have on one simple structure like $\left[\mathrm{Cr}_{3}\left(\mathrm{C}_{3} \mathrm{H}_{5} \mathrm{O}_{2}\right)_{6} \mathrm{O}\left(\mathrm{H}_{2} \mathrm{O}\right)_{3}\right]$ $\mathrm{NO}_{3} \times 0.5 \mathrm{H}_{2} \mathrm{O}$ and what is wrong with publishing questionable (or even unintentionally wrong) results? Should we still describe the structure in a paper if we must supply CIF with all intensity/refinement data? Why one might still consider service crystallographer as a valuable co-author of publication? Let us try to get a consensus on those and some other questions

Keywords: refinement, publication, checkcif
MS20-P04

\section{Shine bright like a diamond: microfocus $\mathrm{X}$-ray sealed tube sources with diamond hybrid anode technology}

Juergen Graf ${ }^{1}$, Tobias Stuerzer ${ }^{2}$, Holger Ott ${ }^{2}$, Paul Radcliffe ${ }^{1}$,

Carsten Michaelsen ${ }^{1}$

1. Incoatec GmbH, Geesthacht, Germany)

2. Bruker AXS GmbH, Karlsruhe, Germany

email: graf@incoatec.de

Diamond exhibits several unique properties, such as high thermal conductivity, low thermal expansion, extreme hardness, chemical inertness and a fairly high transmission coefficient for X-ray radiation. The thermal conductivity of diamond is about 5 times higher than that of copper and the highest known conductivity of all bulk materials [1]. Therefore, diamond, being a non-toxic material, is increasingly replacing traditional materials for the thermal management in challenging applications where a high local heat load needs to be dissipated, such as in heat sinks for high-power microelectronic devices.

The high transmission coefficient, even at low X-ray energies, fosters the use of diamond in X-ray components such as carrier membranes in X-ray lithography or X-ray windows. In X-ray sources, diamond can be used as a transparent heat sink directly coupled to the anode material. This improves the heat dissipation considerably and allows for an increase in tube brilliance by applying a higher power load on the anode.

The I $\mu \mathrm{S}$ DIAMOND is a new type of microfocus sealed tubes using a unique anode technology, the diamond hybrid anode. It takes advantage of the exceptional high thermal conductivity of diamond by using a bulk industrial diamond as a heat sink, which is coated with a thin layer of the target material, such as $\mathrm{Cu}$, Mo or Ag. The diamond heat sink makes the heat dissipation in a diamond hybrid anode more efficient. Consequently, the I $\mu$ S DIAMOND can accept a higher power density in the focal spot on the anode without damaging the surface of the target layer. The balanced heat management in the source assures that the intensity loss over time is only a few percent over $10,000 \mathrm{~h}$ of full power operation, which is significantly lower than the intensity degradation observed for microfocus rotating anodes [2,3]. Therefore, the intensity of the I $\mu$ S DIAMOND is about $20 \%$ higher than the average intensity output of a modern microfocus rotating anode.

The I $\mu$ S DIAMOND establishes a new class of X-ray sources, combining an intensity output that exceeds the average intensity of a microfocus rotating anode with all the comfort and lifetime of a standard microfocus sealed tube with a bulk anode. In this presentation, we will be discussing the main features of the I $\mu$ S DIAMOND and presenting selected results to demonstrate the impact of this new class of microfocus sealed tube X-ray sources on the data quality for applications, such as protein and small molecule crystallography. 
References:

[1] Moore, A.L. \& Shi L. (2014). Materials Today, 17, 163.

[2] Mehranian A., Ay M. R., Riyahi Alam N. \& Zaidi H. (2010), Med Phys., 37, 742-752.

[3] Kákonyi R., Erdélyi M. \& Szabó G. (2010), Med. Phys., 37, $5737-5745$.

Keywords: X-ray Source, Diamond, Instrument Development

\section{MS20-P05}

\section{Integrated crystallographic services at EMBL-Hamburg}

Saravanan Panneerselvam ${ }^{1}$, Gleb Bourenkov ${ }^{1}$, Guillaume Pompidor ${ }^{1}$, Isabel Bento ${ }^{1}$, Johanna Hakanpää ${ }^{1}$, David Von Stetten ${ }^{1}$, Maria Garcia Alai $^{1}$, Thomas R. Schneider ${ }^{1}$

1. EMBL-Hamburg, Hamburg, Germany

email: saravanan.panneerselvam@embl-hamburg.de

EMBL Hamburg is operating three beamlines (P12 for small-angle X-ray scattering 'SAXS', P13 and P14 for macromolecular crystallography ' $\mathrm{MX}$ ') on the PETRA III storage ring at DESY (Hamburg, Germany). The beamlines are embedded in the Integrated Facility for Structural Biology which - in addition to the X-ray experiments on the beamlines - offers facilities and services for sample preparation and characterization and for data analysis.

By using the highly brilliant X-ray beam produced by PETRA III, beamlines P13 and P14 enable X-ray diffraction experiments on the most challenging crystals:

- small $\left(<100 \mu \mathrm{m}^{3}\right)$ crystals can be exploited using micro-focused X-ray beams and serial crystallography strategies

- optimum data can be extracted from large crystals with a highly parallel large beam.

- phasing is facilitated by a high photon flux at low $\mathrm{X}$-ray energies

- diffraction from large unit cells $(>1000 \AA)$ can be resolved

- data from fragile crystals can be collected in situ in CrystalDirect $^{\mathrm{TM}}$ plates

- For pump-probe experiments, a dedicated endstation is under construction on P14.

For less challenging cases, a highly automated and integrated environment is in place for high-throughput data collection.

The Sample Preparation and Characterization facility (SPC) provides an entry point for protein samples which still need to be tested and prepared for use in SAXS and/or MX. A suite of biophysical protocols is available to evaluate the suitability of user-supplied samples; preparative techniques can then be applied to optimize samples for crystallization. For crystallization, high-end equipment is in place that can be accessed remotely via the web-based CRIMS system. A CrystalDirect system has been installed for automated crystal harvesting and a dedicated derivative lab is available for users for derivatization experiments.

Various modes of access are available (For details, please visit: www.embl-hamburg.de):

- competitive: P13 / 14

- collaborative: P13 / P14

- EU-supported (iNext): P13 / P14 / SPC

- fee for service (academic / industrial): P13 / P14 / $\mathrm{SPC}$

We will describe the user-accessible facilities, examples of projects 'from protein to structure', experimental highlights, and the available access modes.

Keywords: beam lines,micro crystallography, integrated facility 